\title{
JVR/ESM Prize 2019 Awarded to Prof. Lydia Sorokin
}

At the recent European Society of Microcirculation (ESM) Conference in Maastricht, Lydia Sorokin was awarded the Journal of Vascular Research (JVR)/ESM Prize. This Prize aims to spotlight research with high impact on vascular biology and the microcirculation with the goal of making an award to one of the most prominent researchers in the field. The editors of $J V R$, together with the scientific committee of the ESM, awarded the prize to Prof. Lydia Sorokin in recognition of her work on vascular matrix proteins with special reference to laminins.

Born in Perth, WA, Australia, Prof. Sorokin graduated in biochemistry and physiology at the University of Western Australia. Supported by a prestigious "Commonwealth Postgraduate Scholarship," she completed her $\mathrm{PhD}$ in 1987 on the topic of "Receptor-Mediated Endocytosis of Transferrin Receptors." Her PhD was unusual in that it resulted in 6 first author papers and proposed, for the first time, the existence of 2 endocytotic pathways, a slow- and a fast-cycling pathway - a concept that was novel at the time and has since become well accepted. Supported by a scholarship from the University of Western Australia and a fellowship from the DAAD (German Academic Exchange Service), she continued her scientific career in Europe, where she first worked as postdoctoral fellow at the Friedrich Miescher Laboratories of the Max Planck Society in Tübingen. It was during this period that she proposed the existence of more than the then

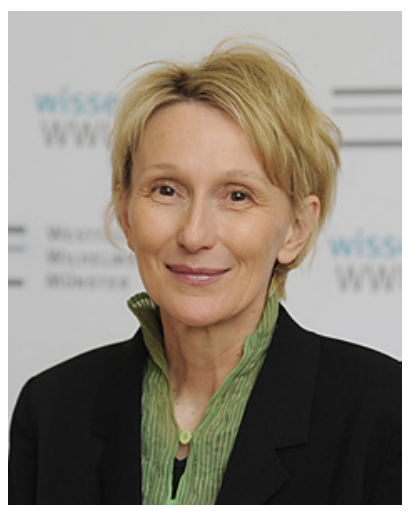

Prof. Lydia Sorokin

known 3 laminin isoforms, in particular in the vasculature - a topic that at the time was not well accepted. She then moved to the University of Erlangen, Germany, where she became first an independent group leader at the age of 30 years and later a junior professor in the Interdisciplinary Center for Clinical Research (IZKF). In 2000, she was awarded the Swedish Natural Science Research Council Senior Research Fellowship, and she moved in 2002 to the Lund University to take up the Chair of Tissue Biology. In 2005, Lydia Sorokin accepted the Chair of Pathobiochemistry at the Medical Faculty of the University of Münster, Germany. Since then she has been a member of many advisory boards of prestigious 
institutions, including the Max Planck Society, has initiated several international graduate schools, and is the spokesperson of the "Cells-in-Motion" Cluster of Excellence at her university. During all these years, she also served as an adjunct professor at her "home university" in Western Australia. She has won several prestigious fellowships and awards.

In the beginning, Lydia Sorokin's work focused on the development of specific tools for the basement membrane (BM) integral proteins, laminins, and the detailed description of the expression of their mRNAs and proteins during development, which provided the first molecular evidence for new laminin isoforms and the identification of 2 of the 5 known laminin a chains, laminin $\alpha 4$ and $\alpha 5$. This work revealed that the role of the $\alpha 4$ - and a5-containing laminins in endothelial BMs were not essential for development and led to a broadening interest in vascular barrier functions, leukocyte extravasation, and in secondary lymphoid organs. Via the generation of a transgenic mouse model, Prof. Sorokin was able to show that laminin $\alpha 5$ acts as a barrier to leukocytes infiltration of the brain and most peripheral tissues. Her work showed for the first time that the blood vessels of the brain are associated with 2 biochemically distinct BMs, differing in laminins but also other extracellular matrix molecules and, more importantly, showing functional differences. In vivo and in vitro studies showed that laminin $\alpha 5$ acts as a stop signal for extravasating leukocytes. These studies identified the so-called perivascular cuff as a confined compartment between the endothelial and parenchymal BMs and highlighted the importance of the parenchymal $\mathrm{BM}$ as a limiting factor in the development of clinical symptoms in neuroinflammation, a structure that had not been recognized by neuroimmunologists because of the lack of tools to identify the 2 BMs.

Lydia Sorokin's group has shown that the gelatinases, MMP-2 and MMP-9, are critical for leukocyte penetration of the parenchymal border - not as general degraders of the BM, as thought to be generally the case for many years, but as focal and selective cleavers of cellular receptors (dystroglycan) and chemokines only at sites of leukocyte penetration. This focal nature of MMP activity at the blood-brain barrier and the correlation between MMP activity and the appearance of clinical symptoms of neuroinflammation has led to the development of new diagnostic imaging strategies to noninvasively identify neuroinflammation in multiple sclerosis patients.

Prof. Sorokin's curiosity in the function of laminins and more generally in BMs has also revealed unique roles for this family of molecules in the reticular fiber networks of lymph nodes and, more recently, in the spleen and Bcell function. New directions in the Sorokin lab in the field of vascular physiology also suggest a role for laminin $a 5$ in the biomechanics of resistance arteries and, hence, their competence to respond to changes in blood flow and pressure.

Her highly innovative work is currently reflected by 133 peer-reviewed papers, 14 peer-reviewed reviews, and 8 book chapters.

At the ESM Meeting, her excellent overview of her current research on the role of laminin $\alpha 5$ in shear response of resistance arteries showed her enthusiasm for science and her excellent teaching capabilities in sharing her work with young scientists.

Ulrich Pohl 